\title{
Uterine and Subendometrial Arteries Doppler in Patients with Recurrent First Trimestric Abortion
}

\author{
Ismail T. El Garhy, Ashraf H. Mohamed, Ahmed S. Sultan* \\ Department of Obstetrics \& Gynecology, Faculty of Medicine, Al-Azhar University \\ *Corresponding author: Ahmed S. Sultan*; Mobile: 01017792934 Email: ahmdsalem103@ gmail.com
}

\begin{abstract}
Background: Unexplained Recurrent miscarriage remains a frustrating problem for the clinician and a distressing condition for the affected couple. Recurrent pregnancy loss is defined as three or more successive spontaneous abortion. The incidence of recurrent pregnancy loss is $1-2 \%$ in the fertile population.

Aim of the Work: This study aimed to find out any difference in uterine artery pulsitility index (PI) between women with history of recurrent unexplained first trimestric abortion and women without this history. Patients and Methods: One hundred cases from Al-Azhar University Hospitals (Al-Hussein and Sayed Galal) were included in the study and classified into two groups: Recurrent pregnancy loss (RPL) group: 50 cases with history of recurrent unexplained abortion. Control group: 50 cases with no history of abortion and one full term child at least. Uterine artery Doppler [Pulsatility Index (PI)] assessment was done to all patients in the study during the luteal phase of spontaneous menstrual cycle.

Results: PI in the RPL group was found to be elevated compared to the control group.

Conclusion: Assessment of uterine perfusion through measurement of uterine artery Doppler (PI) could be of value in cases with recurrent unexplained first trimestric abortion
\end{abstract}

Key Words: Recurrent pregnancy loss - Doppler US - Pulsatility index

\section{Introduction}

Abortion is defined as expulsion or removal of products of conception from the uterus before viability of the fetus or if fetus weight is less than $500 \mathrm{~g}$ or its length is less than $25 \mathrm{~cm}$. Although the true incidence of spontaneous abortion is unknown, approximately $15 \%$ of clinically evident pregnancies and $60 \%$ of chemically evident pregnancies end in spontaneous abortion. Eighty percent $(80 \%)$ of spontaneous abortions occur prior to 12 weeks gestation ${ }^{(1)}$. Moreover, recurrent pregnancy loss is defined as three or more successive spontaneous abortion (2). Conventionally, the American Society of Reproductive Medicine recently redefined recurrent pregnancy loss as two or more failed clinical pregnancies as documented by ultrasonography or histopathological examination ${ }^{(3)}$.

The etiology is often unclear and may be multifactorial, with much controversy regarding diagnosis and treatment. Reasonably accepted etiologic causes include genetics, anatomical, endocrine, placental anomalies, hormonal problems, infections, smoking, alcohol consumption, exposure to environmental risk factors, psychological trauma, stressful life events, certain coagulation conditions and immunoregulatory proteins defects ${ }^{(4)}$.

The incidence of recurrent pregnancy loss is $1-2 \%$ in the fertile population ${ }^{(5)}$. The risk of recurrence increases with the maternal age and number of successive losses. Recurrent pregnancy losses may be attributable to treatable conditions such as hypercoagulable states, autoimmune diseases, endocrine disturbances or maternal anatomic abnormalities, and the high proportion of cases (up to 40-50\%) have unidentifiable causes ${ }^{(6)}$.

Recurrent abortion is best investigated before another pregnancy occurs ${ }^{(5)}$. Uterine receptivity is likely to be regulated by a number of factors including uterine perfusion and is of great importance in achieving a normal pregnancy. Studies suggest that uterine artery perfusion may regulate endometrial receptivity, and that poor uterine perfusion could be one of the causes of unexplained abortions and, probably, of faulty implantation. Before pregnancy, blood flow of the uterine artery demonstrates high resistance to absent or reversed diastolic flow ${ }^{(7)}$. Transvaginal pulsed Doppler ultrasound allows noninvasive evaluation of uterine circulation ${ }^{(8)}$.

Color Doppler is used in obstetrical ultrasound as a complementary tool to gain information about the presence, direction and velocity of blood flow. The pulsatility index (PI) of uterine artery has been known to diminish progressively during the luteal phase during which implantation occurs ${ }^{(8,9)}$.

Therefore, it has been proposed that measurement of uterine artery pulsatility index (PI) in the mid luteal phase of spontaneous cycles 
might isolate patients with recurrent pregnancy loss associated with impaired uterine circulation (7).

\section{Aim of the Work}

To find out any difference in uterine artery pulsitility index (PI) between women with history of recurrent unexplained first trimestric abortion and women without this history

\section{Patients and Methods}

This study (case controlled study) was performed during the period from June 2017 to June 2018. We had 100 of non-pregnant women presented to the Outpatient Clinic Al-Azhar University Hospitals (El-Hussein and Sayed Galal). The women were classified into two groups:

$>$ (Group A): 50 participants presented with a history of idiopathic recurrent pregnancy loss (the RPL group)

$>$ (Group B): 50 participants who had no history of abortion and had at least 1 child born at term (the control group). They presented to the clinic seeking for contraception

At recruitment, the purpose of the study was adequately explained to each participant. All of them were subjected to two dimensional ultrasound examination and Doppler study for measuring the pulsitility index (PI), of uterine arteries.

\section{Inclusion Criteria for the recurrent pregnancy} loss group:

- Three or more successive unexplained first trimestric abortion.

- Age between 20-40 years old.

- Regular menstrual cycles for the previous three cycles before the study.

- No hormonal contraception or intrauterine devices.

- Normal endocrinal status including serum thyroid-stimulating hormone, free thyroxin (T4), glucose tolerance test and progesterone levels between days 19 and 21 of the menstrual cycle.

\section{Inclusion Criteria for the control group:}

- Age between 20-40 years old.

- Regular menstrual cycles for the previous three cycles before the study.

- No hormonal contraception or intrauterine devices.

- Normal obstetric history with at least one previous term pregnancy but no abortions

\section{Exclusion Criteria for both groups:}

- Systemic diseases that might affect the hemodynamic indices e.g. thrombocytopenia, thyroid disease, autoimmune disease cardiovascular disease, DM.etc.

- History of consanguinity.

- Family history of chromosomal abnormalities (e.g. trisomy 21, trisomy 13 , Turner's disease ...etc.).

- Patient age less than 20 years or more than 40 years old.

- Women in the follicular phase or menstrual phase.

- Women having uterine alterations on office hysteroscopy.

- Women having cervical incompetence on transvaginal ultra sonography.

\section{Steps \& Methods:}

All patients recruited gave an informed consent and were subjected to the following:

\section{1- Complete history including:}

- Personal history: their names, age, addresses, occupations, special habits, and history of consanguinity.

- Menstrual history: asking about regularity of cycles frequency, duration and amount of bleeding of each cycle, and date of the last menstrual period.

- Obstetric history: asking about parity and method of previous deliveries, time at which previous abortions had been occurred and whether they had been followed by surgical evacuation or not, and ask about date of the last delivery or abortion.

- Past history: past history of systemic diseases such as diabetes mellitus, hypertension, renal disease, past history of infants with chromosomal abnormalities such as trisomy 21, history of consanguinity, and past history of thyroid troubles.

\section{2- General, abdominal and pelvic examination:}

- General examination: Signs of thyroid disease, autoimmune disease, cardiovasculare disease.etc.

- Breast examination for swelling or nipple discharge

- Abdominal examination:Inspection, palpation and percussion were done to exclude any detectable pathologic lesions e.g. spleenomegaly and hepatomegally. 
- Pelvic examination:-to asses uterine size and the presence or absence of adnexal masses.

\section{Type of ultrasound machine:}

Sonography was performed using ultrasound machine (medisone X6) equipped with vaginal transducer. Transvaginal examination was performed in the second phase of the menstrual cycle, between days 18-23. (due to the endometrium is normally a non receptive environment for an embryo except during implantion window).

implantion window is a period during which the endometrium is optimally receptive to implanting blastocyst.

Implantion of human embryo may occur only during regulated "implantation window" on day $6-10$ post ovulation.

All examination were performed by one sonographer

\section{Technique of examination}

The vaginal probe was covered with an examining glove containing a small amount of gel. The gel ensured good contact between the transducer and the overlying glove. Care was taken to avoid trapping any air bubbles, which might create unwanted artifacts on the screen. Cross infection was prevented by the use of probe cover and disinfectants. With the women lying in the lithotomy position after evacuating her urinary bladder, the transvaginal probe is inserted gently into the vagina and placed in the anterior fornix, and the internal cervical os and the external one are identified, and uterus is examined to asses any uterine anomaly that might interfere with pregnancy such as uterine septum, bicornuate uterus, uterine myomas .....etc.; and to assess and measure the endometrial thickness.

The probe was then moved laterally and the right uterine artery is identified, using color Doppler, as an aliasing vessel running along the side of the cervix at the level of the internal OS, then the left one is identified by the same way.

Pulsed wave Doppler is used to obtain clear, consistent, flow velocity waveforms of both uterine arteries. Pulsatility index (PI) (PI=S$\mathrm{D} /$ mean) is measured bilaterally. The PI reported will be the arithmetic mean for the last three cardiac cycles.

\section{Statistical analysis:}

Data were statistically described in terms of mean \pm standard deviation (SD). Comparison between cases of RPL and control groups was done using Student' $t$ test for independent samples. Correlation between various variables was done using Pearson moment correlation equation for linear relation. $p$ values less than 0.05 was considered statistically significant. All statistical calculations were done using computer programs Microsoft Excel 2010 (Microsoft Corporation, NY, and USA) and SPSS (Statistical Package for the Social Science; SPSS Inc., Chicago, IL, USA) version 15 for Microsoft Windows.

\section{Results}

Table (1): Demographic data of all women in the study

\begin{tabular}{||c|c|c|c||}
\hline Variable & $\begin{array}{c}\text { RPL } \\
\text { group } \\
\text { N=50 }\end{array}$ & $\begin{array}{c}\text { Control } \\
\text { N=50 }\end{array}$ & P value \\
& & & \\
\hline \hline Age(years) & $20: 40$ & $20: 40$ & \\
-Range: & 28.036 & $28.04 \pm$ & \\
-Mean \pm SD: & \pm 4.83 & 5.771 & $0.764(\mathrm{NS})$ \\
\hline Parity & $1: 3$ & $1: 5$ & \\
-Range: & $1.12 \pm$ & $2.62 \pm$ & \\
-Mean \pm SD: & 1.023 & 1.338 & $0.001(\mathrm{~S})$ \\
\hline $\begin{array}{l}\text { Serum Progesterone } \\
\text { (ng/ml) }\end{array}$ & $12: 16$ & $12.5: 17$ & \\
-Range: & $14.0 \pm$ & $14.3 \pm$ & \\
-Mean \pm SD: & 2.7 & 2.4 & $0.002(\mathrm{~S})$ \\
\hline Endometrial & & & \\
thickness (mm) & $7: 11$ & $8: 14$ & \\
-Range: & $9.7 \pm$ & $11.2 \pm$ & \\
-Mean \pm SD: & 2.9 & 2.5 & $.43(\mathrm{NS})$ \\
\hline
\end{tabular}

Table (1) showed that there were no statistically significant differences between RPL group and control group regarding the mean age and endometrial thickness. The mean parity and serum progesterone level of women in the control group was significantly higher than that in the RPL group.

Regarding PI in right uterine artery in RPL group and control group there was a statistically significant difference with mean value $2.505 \pm 0.451$ and $2.248 \pm 0.322$ respectively which reflected increased resistance to blood flow in the right uterine artery in RPL group, as shown in table (2).

Table (2): Comparison of right uterine artery PI between RPL and control groups

\begin{tabular}{||c|c|c|c||}
\hline & $\begin{array}{c}\text { RPL group } \\
\mathrm{n}=50\end{array}$ & $\begin{array}{c}\text { Controls } \\
\mathrm{n}=50\end{array}$ & p value \\
\hline \hline Mean \pm SD & $2.505 \pm 0.451$ & $2.248 \pm 0.322$ & $\begin{array}{c}0.001 \\
(\mathrm{~S})\end{array}$ \\
\hline
\end{tabular}


Comparing PI of left uterine artery in RPL group and control group, revealed a significant difference as shown in table (3), this indicated increased blood flow resistance in left uterine artery in RPL group.

Table (3): Comparison of left uterine artery PI between RPL and control groups

\begin{tabular}{||l|c|c||}
\hline & $\begin{array}{c}\text { RPL group } \\
\mathbf{n = 5 0}\end{array}$ & $\begin{array}{c}\text { Controls } \\
\mathbf{n = 5 0}\end{array}$ \\
\hline Mean \pm SD & $2.521 \pm .397$ & $2.307 \pm .331$ \\
\hline p value & \multicolumn{2}{|c||}{$0.004(\mathrm{~S})$} \\
\hline
\end{tabular}

As regards PI of both right and left uterine arteries in RPL group and control group a statistically significant difference was found as shown in table (4).

Table (4): Comparison of mean uterine artery PI between RPL and control groups

\begin{tabular}{|l|c|c||}
\hline & $\begin{array}{c}\text { RPL group } \\
\mathrm{n}=50\end{array}$ & $\begin{array}{c}\text { Controls } \\
\mathrm{n}=50\end{array}$ \\
\hline Mean \pm SD & $2.513 \pm 0.415$ & $2.278 \pm 0.325$ \\
\hline p value & \multicolumn{2}{|c|}{$0.002(\mathrm{~S})$} \\
\hline \hline
\end{tabular}

As regards vascularity index(VI), it was higher in control group $(2.278 \pm 0.325)$ than in case group $(2.513 \pm 0.415)$ which was statistically significant (P value 0.002).

Table (5): Comparison in (FI) between case and control group.

\begin{tabular}{|l|c|c|c|}
\hline & Controls & Cases & P value \\
\hline \hline Mean & 16.87 & 10.2 & 0.000 \\
\hline Std. Deviation & 3.4 & 1.03 & \\
\hline
\end{tabular}

As regards flow index (FI), it was higher in control group $(16.87 \pm 3.4)$ than in case group $(10.2 \pm 1.03)$ which was statistically significant $(\mathrm{P}$ value 0.000$)$ as shown in table (5).

Table (6): Comparison in (VFI) between case and control group.

\begin{tabular}{|l|c|c|c|}
\hline & Controls & Cases & P value \\
\hline \hline Mean & 0.27 & 0.12 & 0.048 \\
\hline Std. Deviation & 0.11 & 0.08 & \\
\hline
\end{tabular}

As regards vascular flow index (VFI), it was higher in control group $(0.27 \pm 0.11)$ than in case group $(0.12 \pm 0.08)$ which was statisticaly significant ( $\mathrm{P}$ value 0.048$)$.
Table (7): Comparison in endometrial thickness ( $\mathrm{mm}$ ) between case and control group.

\begin{tabular}{||l|c|c|c|}
\hline & Controls & Cases & P value \\
\hline \hline Mean & 9.7 & 9.7 & 0.95 \\
\hline Std. Deviation & 1.4 & 1.8 & \\
\hline \hline
\end{tabular}

Endometrial thickness was $9.7 \mathrm{~mm} \pm 1.8$ $\mathrm{mm}$ in the study group and $9.7 \mathrm{~mm} \pm 1.4 \mathrm{~mm}$ in the control group but the difference was not significant ( $\mathrm{P}$ value 0.95$)$.

Table (8): Comparison in endometrial volume (ml) between case and control group.

\begin{tabular}{||l|c|c|c|}
\hline & Controls & Cases & P value \\
\hline \hline Mean & 2.30 & 2.20 & 0.69 \\
\hline Std. Deviation & 0.50 & 0.52 & \\
\hline
\end{tabular}

Endometrial volume was $2.20 \mathrm{ml} \pm 0.52 \mathrm{ml}$ in the study group and $2.30 \mathrm{ml} \pm 0.50 \mathrm{ml}$ in the control group but the difference was not significant (P value 0.69).

\section{Discussion}

A poor uterine perfusion might be one of the causes of unexplained infertility, however, fewer studies correlates RPL and uterine arteries Doppler flowmetry ${ }^{\left({ }^{(10}\right)}$. A study done by Ziegler et al. (11) concluded that low levels of progesterone during the luteal phase cause the uterine vascular impedance to increase. Therefore, the secretory endometrium was used as an inclusion criterion in women with RPL.

Some studies have been conducted to study uterine artery blood flow in patients with recurrent pregnancy loss, and it was found that elevated uterine arterial impedance is associated with recurrent pregnancy loss ${ }^{(\mathbf{1 2})}$.

Also based on studies from IVF-embryo transfer programmes, impedance of blood flow through the uterine arteries is an indicator of the probability of subsequent pregnancy outcome.

The PI of the uterine arteries had known to decreased progressively during the luteal phase during which implantation occurs ${ }^{(\mathbf{1 0})}$.

Optimum uterine receptivity seems to occur when the mean pulsatility index of both arteries is between 2 and 3 and decreasing significantly the implantation and pregnancy rate when pulsatility is over 3 or 4 , or when diastolic flow is not observed in the Doppler waveform. Inadequate 
blood flow would thus prevent implantation, although optimal uterine perfusion does not always mean pregnancy ${ }^{(13)}$. In addition to this, high uterine resistance was observed in less than $10 \%$ of non-conception cycles, which suggests that this parameter is responsible for failure in implantation in very few cases ${ }^{(\mathbf{1 4})}$.

On the other hand, no statistically significant difference between the PI of the right and left uterine arteries could be found within the groups, just as was reported by Steer et al. ${ }^{(10)}$. Because no difference between the right and left sides could be detected, it seemed possible to confirm that the best way to interpret Doppler data for uterine arteries would be through the mean PI of both sides combined.

About $10-15 \%$ of clinically recognized pregnancies end in spontaneous miscarriage and there is an increasing risk of spontaneous miscarriage with maternal age. Decline in endometrial receptivity which is associated with a decrease in uterine perfusion may play an important role in the decrease of implantation rate with age ${ }^{(15)}$.

In our study, we found that the age of the patients in the recurrent miscarriage group and the control group was not significant $(28.6 \pm 4.6$ years and $26.2 \pm 4.7$ years, respectively $)(p=0.06)$ a finding which is in contrast with previous studies (15).

In the present study, we measured uterine artery PI in the luteal phase of spontaneous cycles. We found that uterine artery PI was significantly higher in the recurrent miscarriage group (2.6 \pm $0.36)$ compared to the control group $(1.7 \pm 0.27)$ $(p=0.000)$. A significant positive correlation was found between UAPI and recurrent miscarriage in the study group $(\mathrm{r}=0.8, \mathrm{p}=0.00)$. This finding is in agreement with many previous studies conducted on patients with recurrent pregnancy loss ${ }^{(12)}$.

Likewise Jirous et al. ${ }^{(16)}$ found increased ovarian and uterine flow impedance in women with RPL than in controls. Also in a study conducted by Lazzarin $\boldsymbol{e t}$ al. ${ }^{(17)}$ on 230 women with RPL and 50 fertile controls, they found that uterine arteries PI values in RPL patients $(2.42 \pm$ 0.79 ) were significantly higher with respect to those found in the control group $(2.08 \pm 0.47)$, and when patients were grouped according to different causes of RPL the highest PI values were found among patients with uterine abnormalities (2.82 \pm 1.0 ), antiphospholipid antibodies syndrome ( 2.70 $\pm 1.1)$, and unexplained RPL $(2.6 \pm 0.7)$. This suggested that an impaired uterine perfusion can negatively influence the reproductive function. So, the authors suggested that these data should be considered of importance as specific therapeutic approaches improving the uterine perfusion may lead to better pregnancy outcome ${ }^{(17)}$.

So, in the completion of this previous work Lazzarin et al. ${ }^{(18)}$ conducted a study on sixty women with unexplained RPL and impaired uterine perfusion to determine the effect of different therapeutic approaches on uterine artery PI in those women, patients were randomly assigned to three different therapeutic regimens; 20 patients received a daily dose of $100 \mathrm{mg}$ of aspirin (LDA); 20 patients were treated with omega-3-fatty acids $4 \mathrm{mg}$ daily; and 20 patients received LDA and omega-3-fatty acids, they found that all therapeutic regimens induced an improvement in uterine perfusion with a significant reduction in uterine artery PI values. But they recommended further studies to ascertain whether such improvement in uterine perfusion can effectively lead to better pregnancy outcome in these women ${ }^{(18)}$.

In our study, there was no significant difference between endometrial thickness and endometrial volume between the two groups, a finding which is in agreement with previous studies (16).

It has been proposed to consider the endometrial and subendometrial area as a whole when the uterine perfusion is assessed by colour Doppler, since there is no difference between the endometrial and subendometrial blood flow with respect to the possibility of achieving pregnancy ${ }^{(\mathbf{1 9})}$. In our present study, we evaluated the role of subendometrial vascularity in patients with history of recurrent miscarriage during natural unstimulated cycles, and we found significantly higher subendometrial indices in the control group compared to recurrent miscarriage group, a significant negative correlation was found between VI $(r=-0.56, p-0.00)$, and FI $(r=-0.79$, $p=0.00)$ and recurrent miscarriage in the study group.

Several studies had been conducted to evaluate the role of endometrial and subendometrial vascularity measured by 3D Doppler ultrasound in the prediction of live birth following IVF cycles ${ }^{(\mathbf{1 9}, 20)}$.

Comparable to our results, study had been performed by Vaquero et al. ${ }^{\text {(21) }}$ on 40 women with unexplained RPL and it was found that twenty patients showed increased resistance to uterine arteries blood flow whereas there was normal resistance in twenty cases. The patients with increased resistance to uterine arteries blood 
flow showed lower 3D vascularisation indices . Also, Abdel Wahab et al. ${ }^{(22)}$ evaluated uterine artery blood flow and subendometrial blood flow in women with unexplained RPL compared to normal fertile women. Women were divided into two groups: those with history of unexplained RPL (study group, no. $=40$ ), and those with no history of miscarriage and at least one child was bom at term (control group, no. $=40$ ). Uterine arteries (UA) PI was significantly higher in the study group $(p=$ $0.000)$, on the other hand VI $(p=0.000)$, FI $(p=$ $0.000)$, VFI $(p=0.000)$ were significantly lower in the study group. A significant positive correlation was found between UAPI and recurrent miscarriage in the study group $(r=0.8, p=0.00)$, whereas a significant negative correlation was found between $\mathrm{VI}(r=-0.56, p=0.00)$, and FI $(r=-0.79, / ?=0.00)$ and recurrent miscarriage in the study group. But, no significant correlation was found between endometrial thickness and endometrial volume between the two groups.

Also El-Mazny et al. ${ }^{(12)}$ evaluate uterine artery blood flow using pulsed Doppler, and endometrial and subendometrial microvascularization using (3D) power Doppler, in women with unexplained infertility. In a prospective clinical trial at a university teaching hospital, 40 women with unexplained infertility were compared to 40 fertile parous controls. In the mid-luteal (periimplantation) phase, the endometrial thickness and volume, uterine artery pulsatility index (PI) and resistance index (RI), endometrial and subendometrial 3D power Doppler vascularization index (VI), flow index (FI), and vascularization flow index (VFI), and serum estradiol and progesterone levels were measured in both groups. The uterine artery PI $(\mathrm{P}=0.003)$ and $\mathrm{RI}(\mathrm{P}=0.007)$ were significantly increased and the endometrial VI $(\mathrm{P}=$ 0.029), FI $(P=0.031)$, and VFI $(P=0.001)$ and subendometrial VI $(\mathrm{P}=0.032)$, FI $(\mathrm{P}=0.040)$, and VFI $(P=0.005)$ were significantly decreased in the unexplained infertility group. The endometrial thickness and volume and serum estradiol and progesterone levels, however, were not significantly different between the two groups. They concluded that Peri- implantation endometrial perfusion is impaired in women with unexplained infertility. So, Doppler study of uterine hemodynamics should therefore be considered in infertility work-up.

Also in IVF cycles failure of implantation was associated with absent subendometrial blood flow Zaidi et $\boldsymbol{a l}$. ${ }^{(23)}$, and pregnant patients with live birth had significantly higher endometrial and subendometrial vascularity when compared to those with miscarriage. The absence of colour mapping at the endometrium and subendometrial myometrium means absolute implantation failure Zaidi et al. ${ }^{(23)}$ or significant decrease Chien et al. (19) of the implantation rate. In 75 IVF cycles, Schild et al. (24) showed that all 3D subendometrial power Doppler flow indices performed on the first day of ovarian stimulation. The subendometrial FI was the strongest predictive factor for the outcome among the tested sonographic parameters in pregnant cycles than nonpregnant ones. Kupesic et al. ${ }^{(25)}$ found in 89 patients that subendometrial FI on the day of ET was significantly higher in pregnant cycles, whereas subendometrial VI and VFI were similar between pregnant and nonpregnant patients. $\mathbf{W u}$ et al. ${ }^{(26)}$ also demonstrated in 54 patients that subendometrial VFI on the day of HCG was significantly higher in the pregnant group and was superior to endometrial volume, subendometrial VI and FI in predicting the outcome.

Although, their main objective was to link these results to uterine receptivity in order to create the perfect environment for embryos to implant and increase the chances of couples having successful IVF treatment. Unfortunately most of the works contradicted themselves in one way or another and has still left us uncertain of the relationship between uterine receptivity and endometrium and subendometrium changes. The variations in the day of the ultrasound, patient characteristics, ovarian stimulation and techniques used were the likely reasons that the results differed so much. A standard protocol for this 3D examination is required to allow future studies to be able to eventually state whether this procedure helps to increase pregnancy rates in IVF patients. Until then the value of 3D ultrasound in assessing the endometrium is limited for the predication of pregnancy and should be used cautiously ${ }^{(27)}$.

In our study, the application of binary Logistic regression analysis model found that adding variables has not significantly increased our ability to predict the occurrence of miscarriage. This finding can be attributed to small sample size despite the high significant difference observed between the two groups.

In a study by $\mathbf{N g}$ et al. ${ }^{(28)}$, they found that only endometrial VI was significantly associated with the chance of live birth with an OR of 1.384 $(95 \% \mathrm{Cl}: 1.025-1.869, p=0.034)$. Other parameters were not predictive of live birth.

Despite these findings we could not find any cut off values that could predict the occurrence of miscarriage which may be attributed to small sample size and short period of follow up and the study is limted to pateitent with 
normal uterine cavity by hysteroscopy, women age $(20-40 y)$, day $(18-23)$ of regular cycle, normal thyroid function.

so larger prospective studies are needed aiming to confirm such results and reaching values that can accurately predict such cases and a new method as three dimensional poueer Doppler angiography in the filed of vascular quantification with further recommendation. Emotional support and careful monitoring during early pregnancy help to improve pregnancy outcomes. Empiric treatments with exogenous progesterone or low dose aspirin in women with unexplained recurrent pregnancy loss have no proven value ${ }^{(29)}$.

\section{Conclusion}

The study revealed that the mean uterine artery (PI) value in RPL group was higher than control group $(2.513 \pm 0.415)$ than in the control group $(2.278 \pm 0.325)$ and this reflect increased impedance to blood flow in uterine arteries in women with history of RPL

\section{Recommendations}

- Based on the findings of this study, it is possible to propose the use of transvaginal ultrasonography with Doppler flowmetry in the second phase of the menstrual cycle to assess uterine artery blood flow in women at risk for spontaneous abortion.

\section{References}

1. Chauleur C, Galanaud JP, Alonso S, CocheryNouvellon E, Balducchi JP et al. (2010): Observational study of pregnant women with a previous spontaneous abortion before the 10th gestation week with and without antiphospholipid antibodies. J Thromb Haemost., 8: 699-706.

2. Oliver-Williams CT, Steer PJ (2015): Racial variation in the number of spontaneous abortions before a first successful pregnancy, and effects on subsequent pregnancies. Int $\mathbf{J}$ Gynaecol Obstet., 129: 207-212.

3. Birminghan Y, Alabama T (2013): American Society for Reproductive Medicine Definitions of infertility and recurrent pregnancy loss. Fertil Steril., 99 (1): 63-71.

4. Losos JB, Raven PH, Johnson Y (2013): Biology. New York: McGraw-Hill. Pp: 120709. ISBN 0-07-303120-8

5.

https://onlinelibrary.wiley.com/doi/abs/10.11 11/tog.12009
6. Agarwal K, Alfirevic Z (2012): Pregnancy loss after chorionic villus sampling and genetic amniocentesis in twin pregnancies: a systematic review.Ultrasound in Obstetrics \& Gynecology, 40 (2): 128-34.

7. Guedes-Martins L, Cunha, Gaio R, Macedo F, Almeida $H$ (2014): Internal iliac and uterine arteries Doppler ultrasound in the assessment of normotensive and chronic hypertensive pregnant women. Sci Rep., 4: 3785

8. Casikar I, Lu C, Oates J, Bignardi T, Alhamdan D, Condous G (2012): The use of power Doppler colour scoring to predict successful expectant management in women with an incomplete miscarriage. Hum Reprod., 27: 669-75.

9. Shimamoto K, Sakuma S, Ishigaki $T$ and Makino N (2008): Intramural blood flow: evaluation with color Doppler echography, Radiology, 165 (3): 683-685.

10. Steer CV, Tan SL and Dillon D (1995): Vaginal color Doppler assessment of uterine artery impedance correlates with immunohistochemical markers of endometrial receptivity required for the implantation of an embryo. Fertil Steril., 63: 101-108

11. De Ziegler D, Frydman R (1990): Different implantation rates after transfers of cryopreserved embryos originating from donated oocytes or from regular IVF-ET. Fertile Steril., 54: 682-8.

12. El-Mazny A, Abou-Salem N, Elshenoufy $H$ (2013): Doppler study of uterine hemodynamics in women with unexplained infertility. Eur J Obstet Gynecol ReprodBiol., 171 (1): 84-7.

13. Deichert $U$, Albrand-Thielman $C$, van de Sandt M (1996): Doppler- sonographic pelvic blood flow measurements and their prognostic value in terms of luteal phase and implantation. Hum Reprod., 11: 1591-1593.

14. Cacciatore B, Simberg $\mathbf{N}$, Fusaro $P$ (1996): Transvaginal Doppler study of uterine artery blood flow in in vitro fertilization embryo transfer cycles.EF/7 Steril., 66: 130134.

15. Nybo Andersen A.M, Wohlfahrt J, Christens P, Olsen J, Melbye M (2000): Maternal age and fetal loss: population based register linkage study. BMJ., 320: 1708-1712.

16. Jirous J, Diejomaoh M, Al Othman S, Al Abdulhadi F, Al Marzouk N, Sugathan T (2001): A correlation of the uterine and ovarian blood flow with parity of nonpregnant women having a history of recurrent spontaneous abortion. Gynecol Obstet Invest., 52: 51-54. 
17. Lazzarin N, Vaquero E, Exacoustos C, Romanini E, Amadio A, Arduini D (2007): Mid luteal phase Doppler assessment of uterine artery blood flow in non-pregnant women having a history of recurrent spontaneous abortions: correlation to different etiologies. Fertil Steril., 87 (6): 1383-7.

18. Lazzarin N, Vaquero E, Exacoustos C, Bertonotti E, Romanini E (2009): Low dose aspirin and omega- 3 fatty acids improve uterine artery blood flow velocity in women with recurrent miscarriage due to impaired uterine perfusion. Fert Steril., 92: 296-300.

19. Chien LW, Au HK, Chen PL, Xiao J, Tzeng CR (2002): Assessment of uterine receptivity by the endometrial sub endometrial blood flow distribution pattern in women undergoing in vitro fertilization embryo transfer. Fert Steril., 78: $245-51$.

20. Dechaud H, Bessueille E, Bousquet PJ, Revfmaml L, Hedon B (2008): Optimal timing of ultrasonographic and Doppler evaluation of uterine receptivity to implantation. Reprod Biomed., 16: 368-75.

21. Vaquero E, Lazzarine $N$, Exacoustos $C$, Romanini E, Bertonotti E (2008): Recurrent miscarriage: three dimensional power Doppler Evaluation of endometrial and sub endometrial volume and Vascularity and correlation to uterine arteries blood flow. Ultrasound Obstet Gynecol., 32: 262-6.

22. Hala Abdel Wahab, Doaa Salah El-Din, Eman Zain, Mohamed Abdelgany, Mohamed A.F.M. Youssef (2011): Uterine artery Doppler and subendometrial blood flow in patients with unexplained recurrent miscarriage. Middle East Fertility Society Journal, 16 (3): 209-214.
23. Zaidi J, Campbell S, Pittrof R, Tan S (1995): Endometrial thickness, morphology, vascular penetration, and velocimetry in predicting implantation in an in vitro fertilization program. Ultrasound Obstet Gynecol., 6: 191-198.

24. Schild RL, Holthaus S, d'Alquen J, Fimmers $R$, Dorn $C$, van Der Ven H, Hansmann $M$ (2000): Quantitative assessment of subendometrial blood flow by threedimensional-ultrasound is an important predictive factor of implantation in an in-vitro fertilization programme. Hum Reprod., 15 (1): 89-94.

25. Kupesic S, Bekavac I, Bjelos D, Kurjak A (2001): Assessment of endometrial receptivity by transvaginal color Doppler and threedimensional power Doppler ultrasonography in patients undergoing in vitro fertilization procedures. J Ultrasound Med., 202: 125-34.

26. Wu H, Chiang $C$, Haung $H$ et al. (2003): Detection of the subendometrial vascularization flow index by three dimensional ultrasound may be useful in predicting the pregnancy rate for patients undergoing in vitro fertilizationembryo transfer. FertilSteril., 79: 507-11.

27. Alcazar JL (2006): Three-dimensional ultrasound assessment of endometrial receptivity: a review. Reprod Biol Endocrinol., 4: 56.

28. Ng E H Y, Chan C C W, Tang O S, Yeung W $S$ B, Ho P C (2007): Endometrial and subendometrial vascularity is higher in pregnant patients with live birth following ART than in those who suffer a miscarriage. Hum Reprod., 22: 1134-1141.

29. Coomarasamy A, Truchanowicz EG, Rai $R$ (2011): Does first trimester progesterone prophylaxis increase the live birth rate in women with unexplained recurrent miscarriages? BMJ., 342: d1914. 